\title{
The Definitive Guide to SUSE Linux Enterprise Server 12
}

Sander van Vugt 


\section{The Definitive Guide to SUSE Linux Enterprise Server 12}

Copyright $\odot 2014$ by Sander van Vugt

This work is subject to copyright. All rights are reserved by the Publisher, whether the whole or part of the material is concerned, specifically the rights of translation, reprinting, reuse of illustrations, recitation, broadcasting, reproduction on microfilms or in any other physical way, and transmission or information storage and retrieval, electronic adaptation, computer software, or by similar or dissimilar methodology now known or hereafter developed. Exempted from this legal reservation are brief excerpts in connection with reviews or scholarly analysis or material supplied specifically for the purpose of being entered and executed on a computer system, for exclusive use by the purchaser of the work. Duplication of this publication or parts thereof is permitted only under the provisions of the Copyright Law of the Publisher's location, in its current version, and permission for use must always be obtained from Springer. Permissions for use may be obtained through RightsLink at the Copyright Clearance Center. Violations are liable to prosecution under the respective Copyright Law.

ISBN-13 (pbk): 978-1-4302-6821-5

ISBN-13 (electronic): 978-1-4302-6820-8

Trademarked names, logos, and images may appear in this book. Rather than use a trademark symbol with every occurrence of a trademarked name, logo, or image, we use the names, logos, and images only in an editorial fashion and to the benefit of the trademark owner, with no intention of infringement of the trademark.

The use in this publication of trade names, trademarks, service marks, and similar terms, even if they are not identified as such, is not to be taken as an expression of opinion as to whether or not they are subject to proprietary rights.

While the advice and information in this book are believed to be true and accurate at the date of publication, neither the author nor the editors nor the publisher can accept any legal responsibility for any errors or omissions that may be made. The publisher makes no warranty, express or implied, with respect to the material contained herein.

Managing Director: Welmoed Spahr

Lead Editor: Michelle Lowman

Production Editor: Douglas Pundick

Technical Reviewer: Stewart Watkiss

Editorial Board: Steve Anglin, Mark Beckner, Ewan Buckingham, Gary Cornell, Louise Corrigan, Jim DeWolf, Jonathan Gennick, Robert Hutchinson, Michelle Lowman, James Markham, Matthew Moodie, Jeff Olson, Jeffrey Pepper, Douglas Pundick, Ben Renow-Clarke, Dominic Shakeshaft, Gwenan Spearing, Matt Wade, Steve Weiss

Coordinating Editor: Kevin Walter

Copy Editor: Michael G. Laraque

Compositor: SPi Global

Indexer: SPi Global

Artist: SPi Global

Cover Designer: Anna Ishchenko

Distributed to the book trade worldwide by Springer Science+Business Media New York, 233 Spring Street, 6th Floor, New York, NY 10013. Phone 1-800-SPRINGER, fax (201) 348-4505, e-mail orders-ny@springer-sbm.com, or visit wWw. springeronline. com. Apress Media, LLC is a California LLC and the sole member (owner) is Springer Science + Business Media Finance Inc (SSBM Finance Inc). SSBM Finance Inc is a Delaware corporation.

For information on translations, please e-mail rights@apress.com, or visit wWw. apress.com.

Apress and friends of ED books may be purchased in bulk for academic, corporate, or promotional use. eBook versions and licenses are also available for most titles. For more information, reference our Special Bulk Sales-eBook Licensing web page at www. apress.com/bulk-sales.

Any source code or other supplementary material referenced by the author in this text is available to readers at WWW. apress.com. For detailed information about how to locate your book's source code, go to wWw. apress.com/source-code. 
This book is dedicated to Alex. It's the joy in your eyes that inspires me to do great things in my life. 



\section{Contents at a Glance}

About the Author .........................................................................................

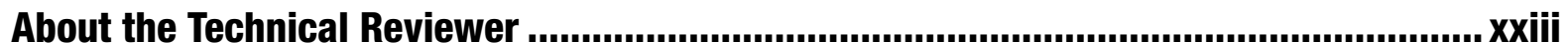

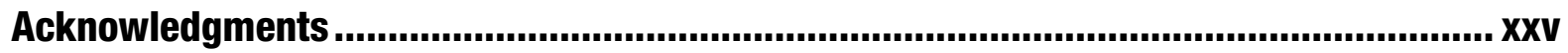

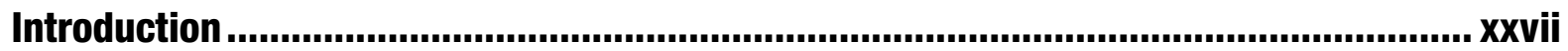

Part I: Basic Skills .................................................................................. 1

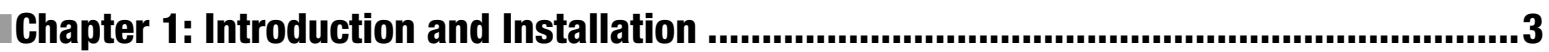

Chapter 2: Basic Skills ...............................................................................................33

Part II: Administering SUSE Linux Enteprise Server ............................ 49

Chapter 3: Managing Disk Layout and File Systems ..................................................51

Chapter 4: User and Permission Management ..........................................................81

Chapter 5: Common Administration Tasks ................................................................99

Chapter 6: Hardening SUSE LinuX ......................................................................131

Chapter 7: Managing Virtualization on SLES ...........................................................161

Chapter 8: Managing Hardware, the Kernel, and the Boot Procedure ..........................177

Part III: Networking SUSE Linux Enterprise Server ........................... 197

Chapter 9: Configuring Network Access................................................................199

Chapter 10: Securing Internet Services: Certificates and SUSE Firewall ....................229

Chapter 11: Basic Network Services: xinetd, NTP, DNS, DHCP, and LDAP ...................259

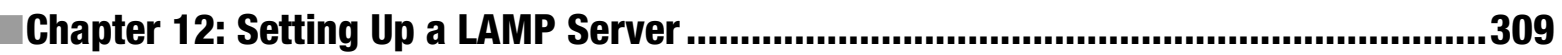

Chapter 13: File Sharing: NFS, FTP, and Samba ..........................................................331 
Part IV: Advanced SUSE Linux Enterprise Server Administration............. 359

Chapter 14: Introduction to Bash Shell Scripting ...................................................361

Chapter 15: Performance Monitoring and Optimizing ..............................................389

Chapter 16: Creating a Cluster on SUSE Linux Enterprise Server.................................433

Chapter 17: Creating a SLES 12 Installation Server ...............................................471

Chapter 18: Managing SUSE Linux .......................................................................

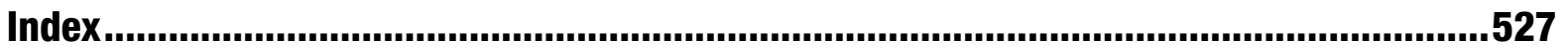




\section{Contents}

About the Author $x x i$

About the Technical Reviewer xxiii

Acknowledgments . XXV

Introduction xxvii

\section{Part I: Basic Skills}

Chapter 1: Introduction and Installation 3

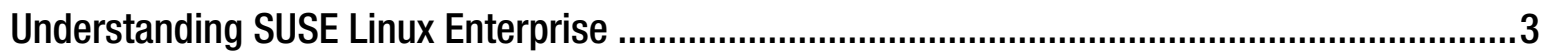

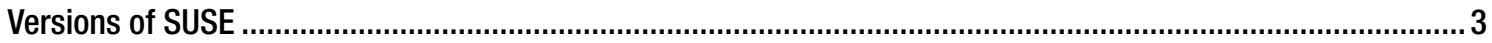

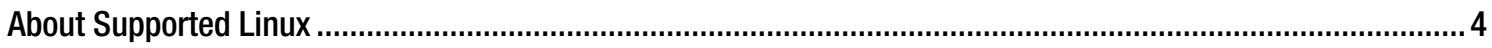

Working with SUSE Linux Enterprise 12 Modules .............................................................. 4

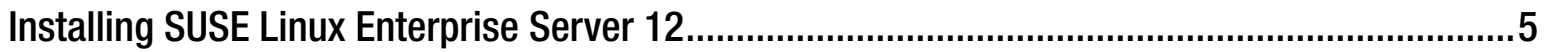

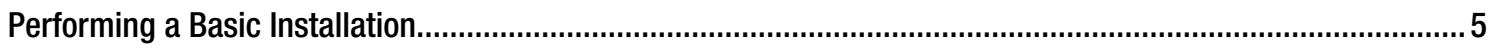

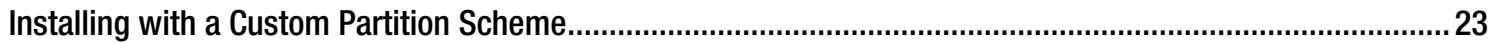

Summary

Chapter 2: Basic Skills .....................................................................................33

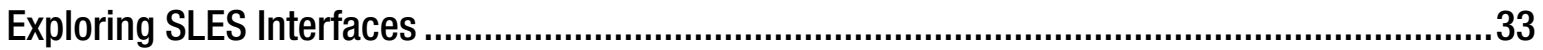

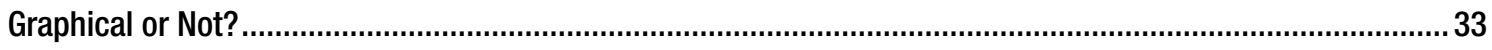

GNOME or KDE?

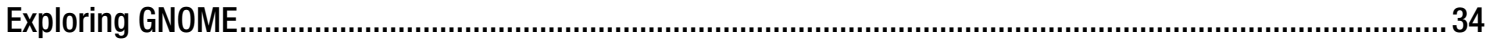

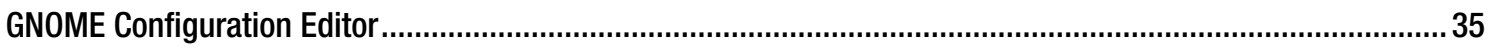

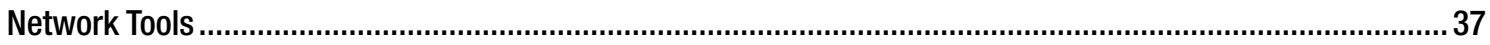

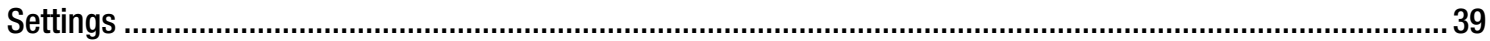




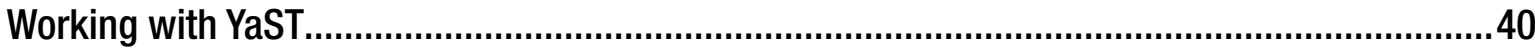

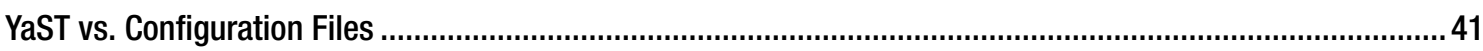

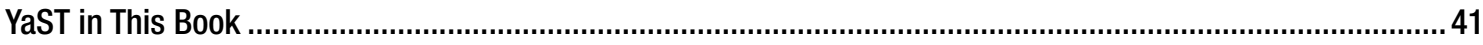

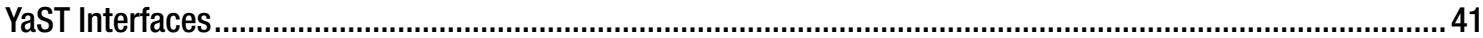

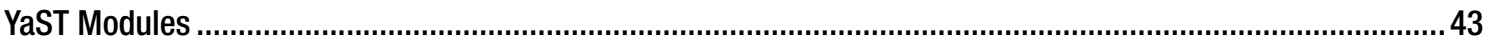

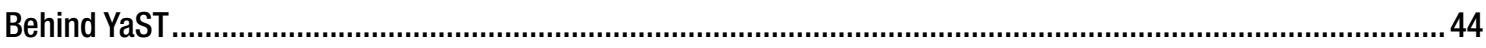

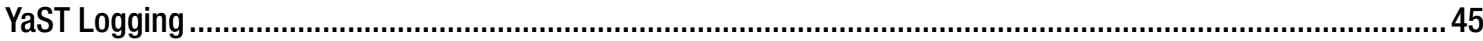

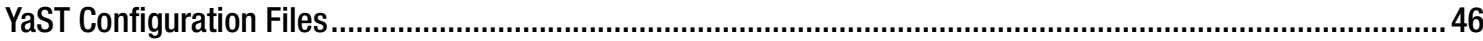

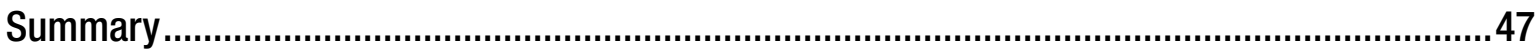

Part II: Administering SUSE Linux Enteprise Server .................................. 49

Chapter 3: Managing Disk Layout and File Systems ...................................................51

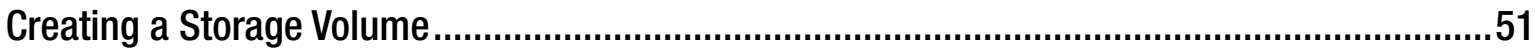

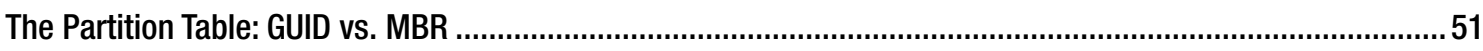

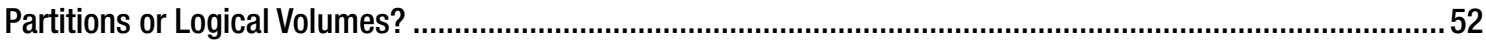

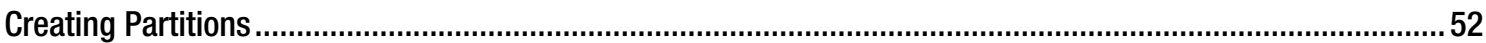

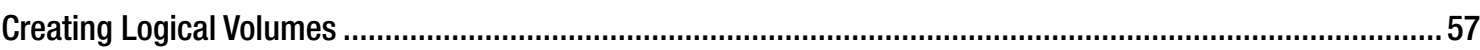

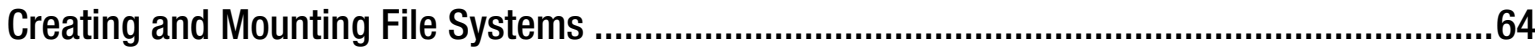

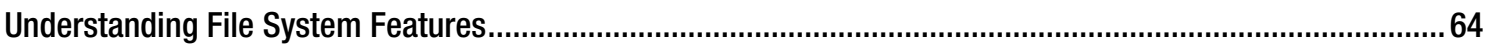

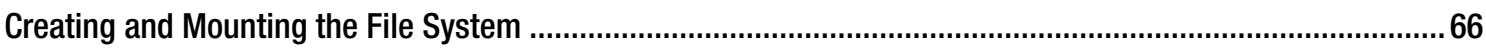

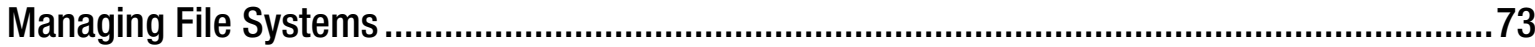

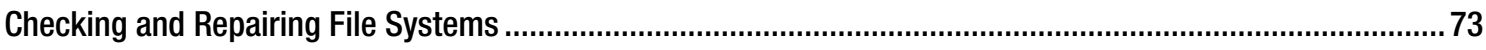

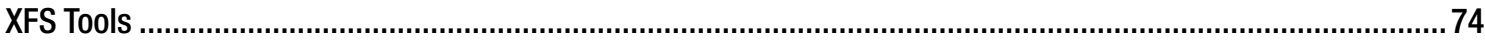

Btrfs Tools and Features ................................................................................................................. 74

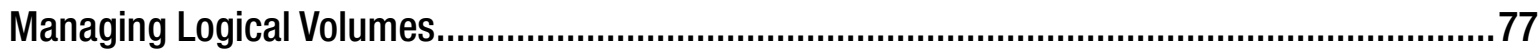

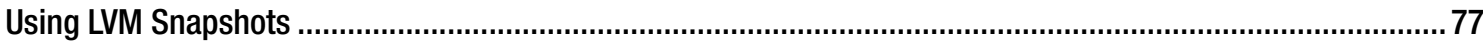

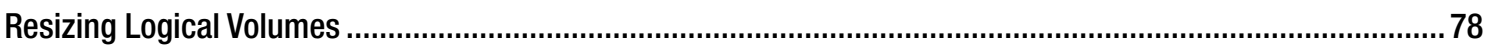

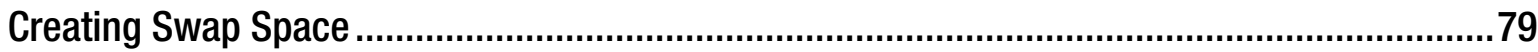

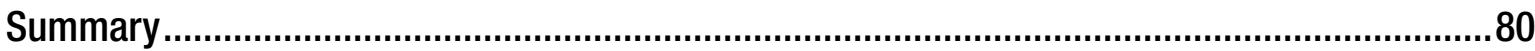


Chapter 4: User and Permission Management

Creating and Managing User Accounts

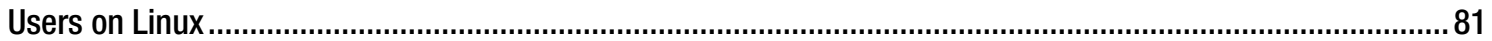

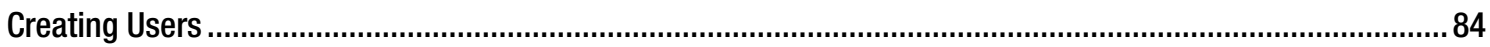

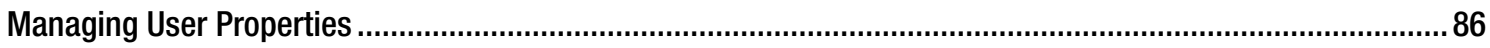

Configuration Files for User Management Defaults .................................................................................. 86

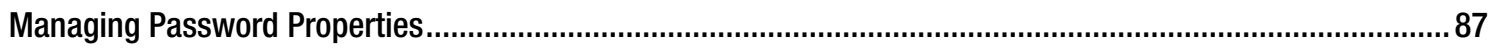

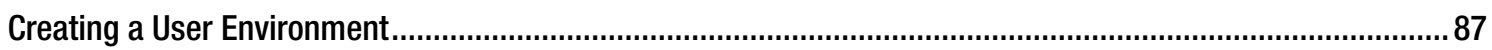

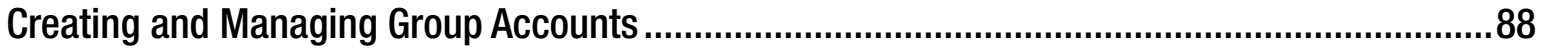

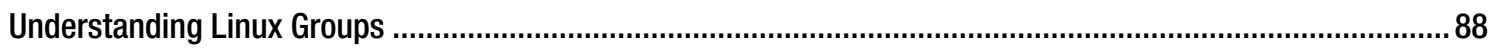

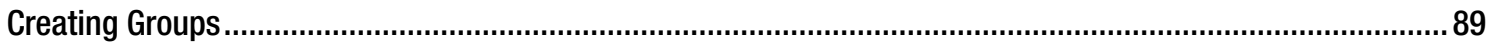

Managing Group Properties............................................................................................................ 90

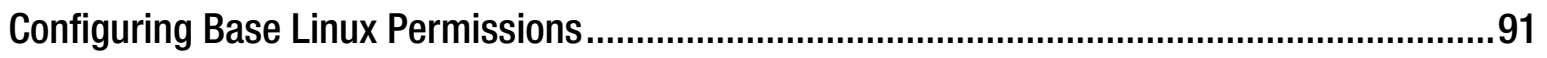

Understanding File Ownership .......................................................................................................91

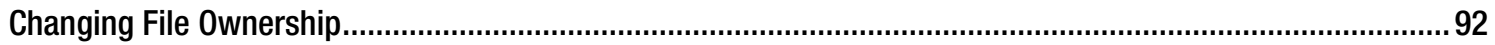

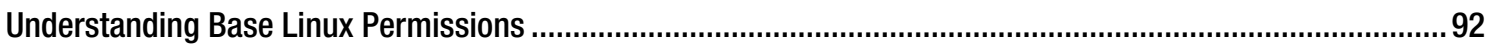

Applying Base Linux Permissions.....................................................................................................

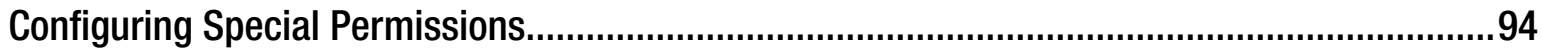

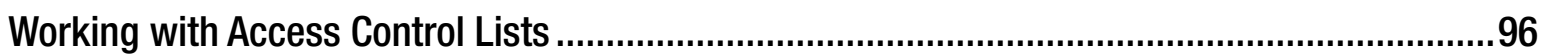

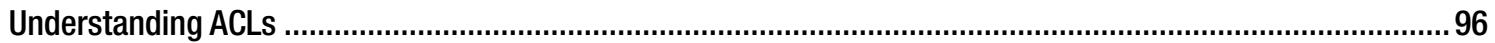

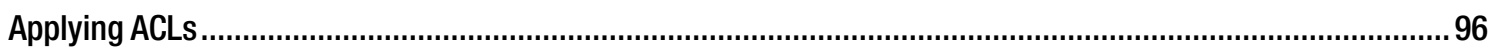

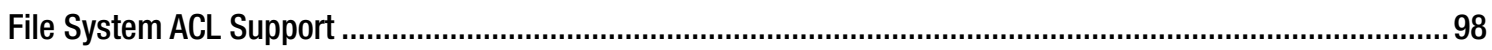

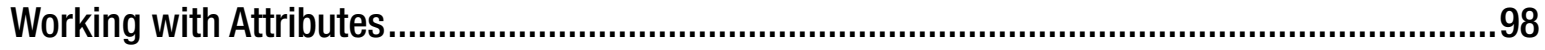

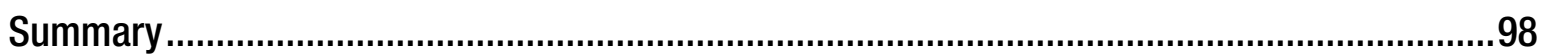

Chapter 5: Common Administration Tasks ....................................................................99

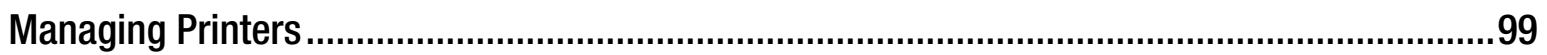

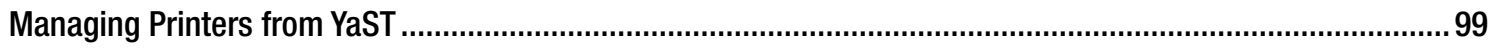

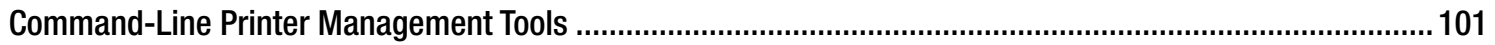




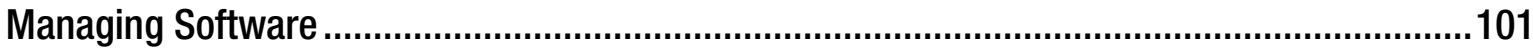

Understanding Repositories and Meta Package Handlers......................................................................... 101

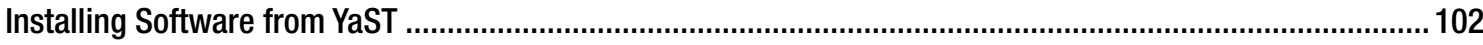

Installing Software from the Command Line ......................................................................................... 109

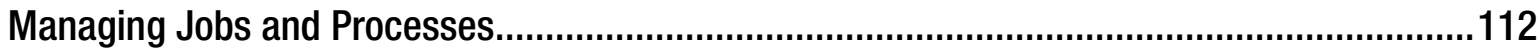

System and Process Monitoring and Management.................................................................................114

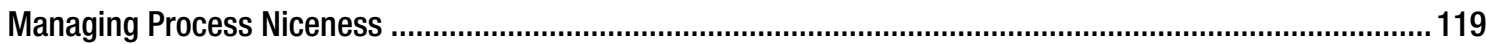

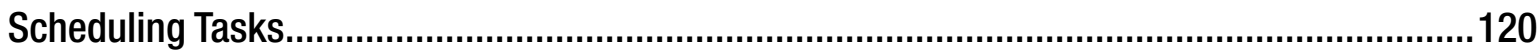

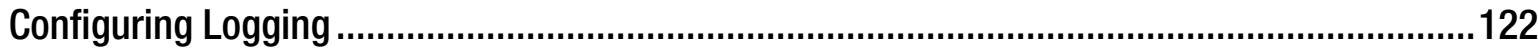

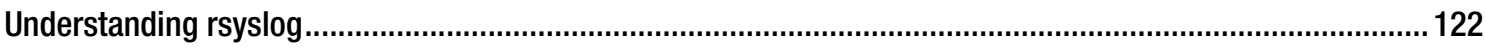

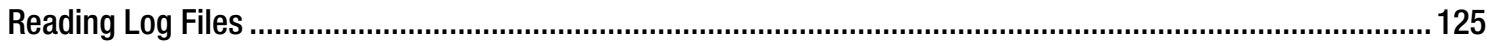

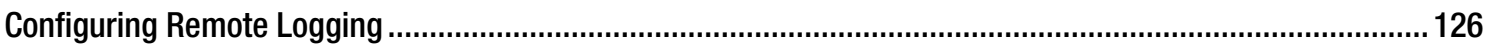

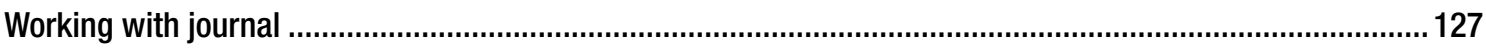

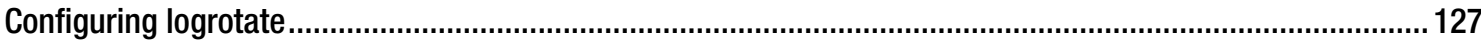

Summary

Chapter 6: Hardening SUSE Linux.............................................................................

Using the YaST Security Center and Hardening................................................................131

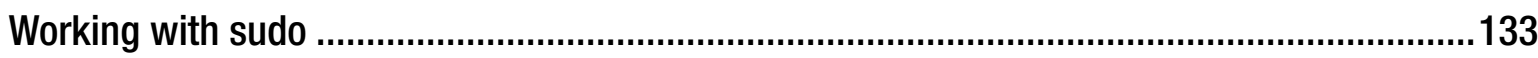

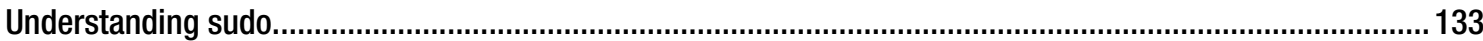

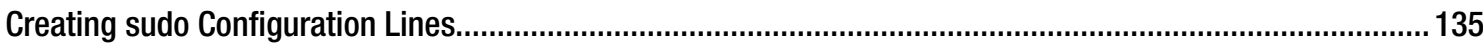

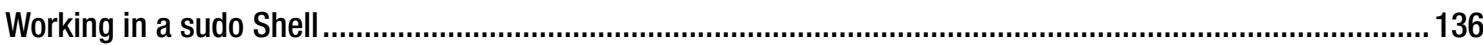

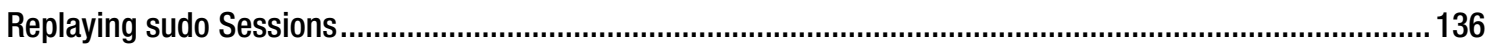

The Linux Audit Framework.................................................................................................137

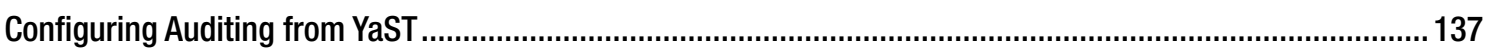

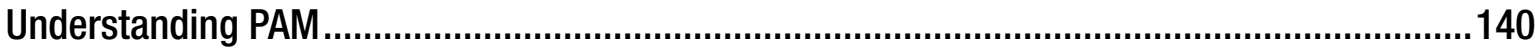

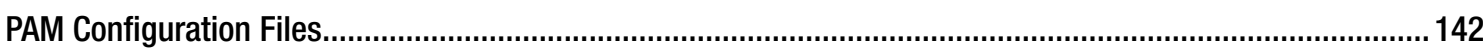

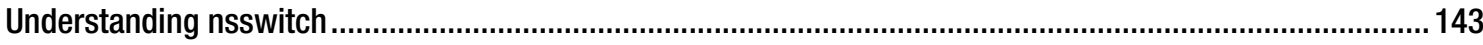

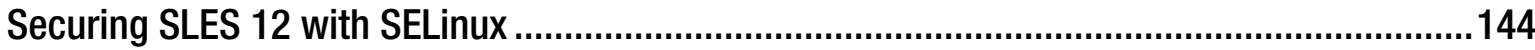

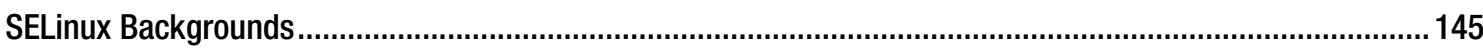

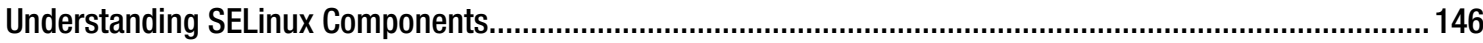


The Policy 146

Installing SELinux on SUSE Linux Enterprise 12 FCS

Configuring SELinux 149

Managing SELinux 151

Troubleshooting SELinux 156

Switching to Enforcing Mode. 158

Summary 159

Chapter 7: Managing Virtualization on SLES

Understanding Linux Virtualization Solutions ..................................................................161

Understanding the KVM Environment...........................................................................161

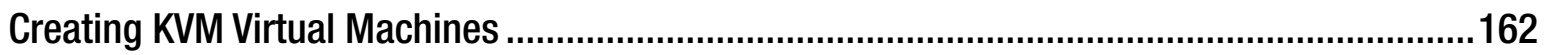

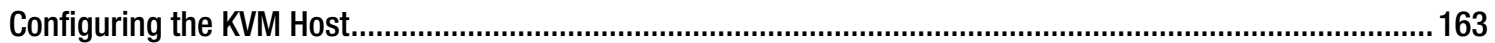

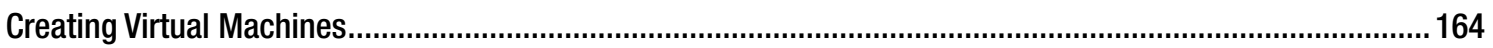

Managing KVM Virtual Machines.................................................................................168

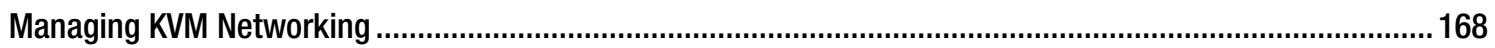

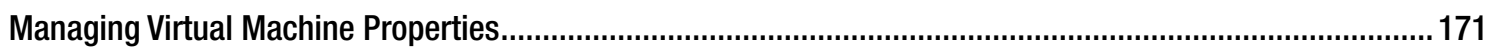

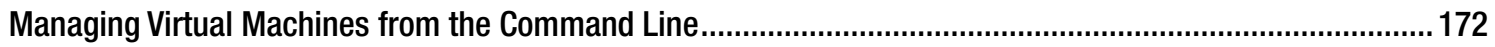

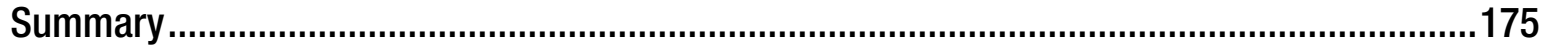

Chapter 8: Managing Hardware, the Kernel, and the Boot Procedure ...........................177

Managing the Linux Kernel ......................................................................................177

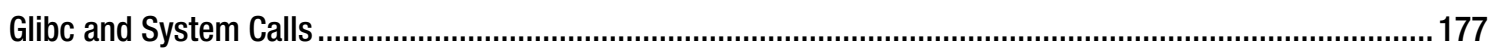

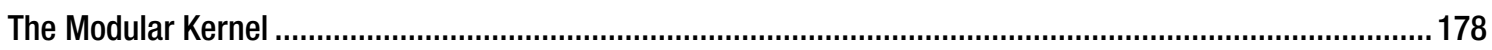

Managing Hardware ............................................................................................181

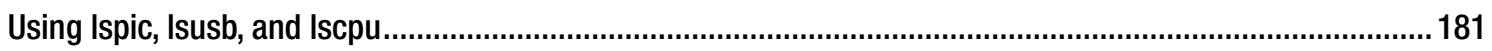

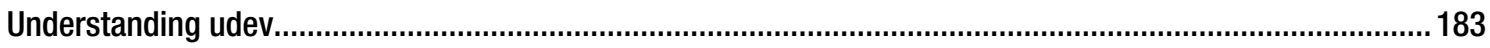

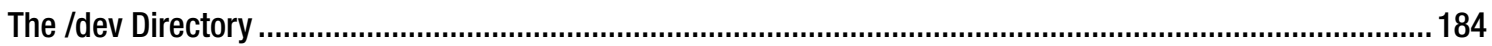

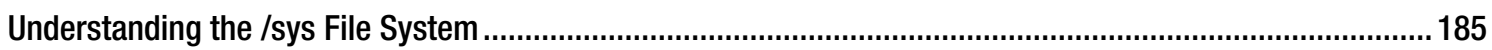

Managing the Boot Procedure.................................................................................... 186

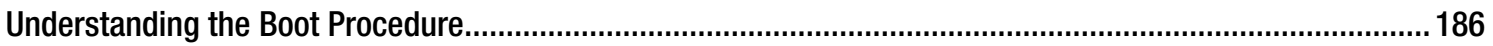

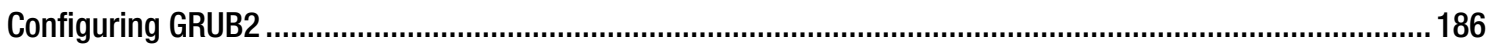


Starting systemd

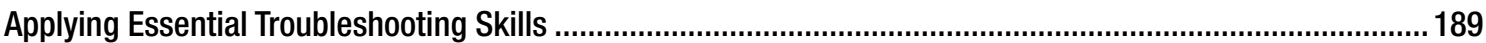

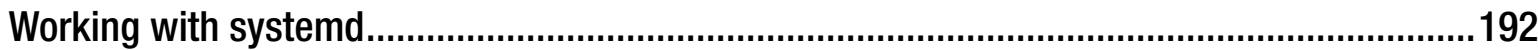

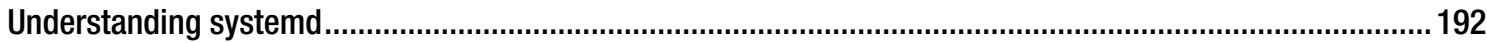

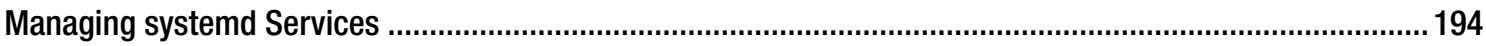

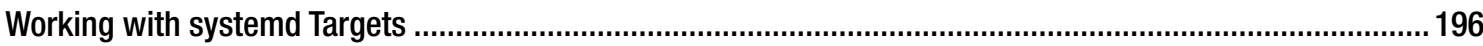

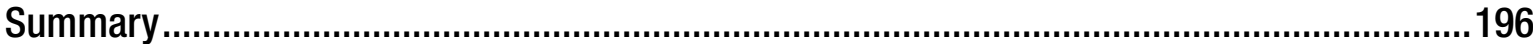

Part III: Networking SUSE Linux Enterprise Server ................................. 197

Chapter 9: Configuring Network Access...............................................................199

Manual NIC Configuration...........................................................................................199

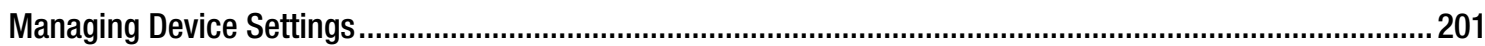

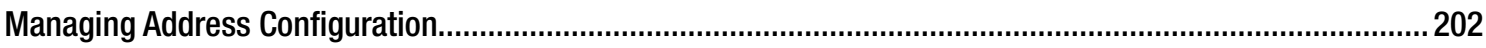

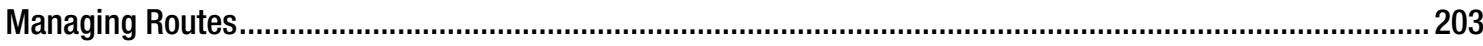

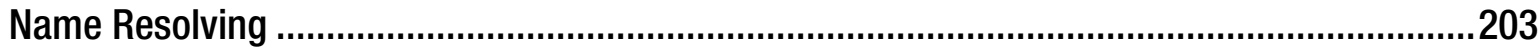

Understanding Wicked .............................................................................................204

Using YaST for NIC Configuration .............................................................................206

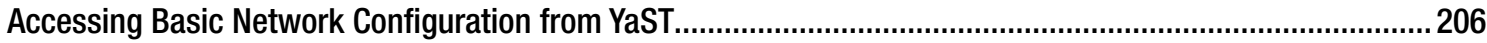

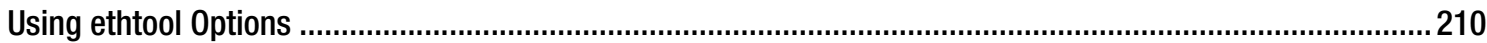

Changing Routing and Name Resolution from YaST .............................................................................. 212

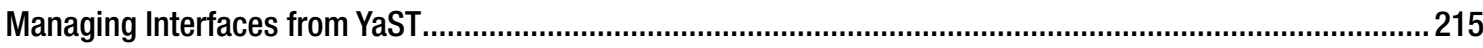

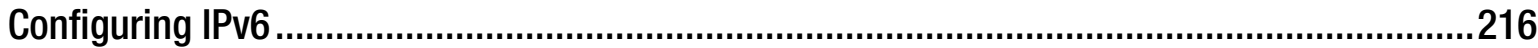

Troubleshooting Networking ...................................................................................217

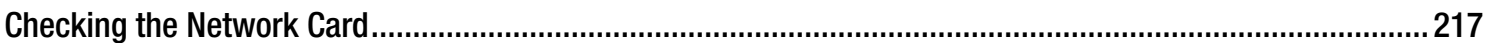

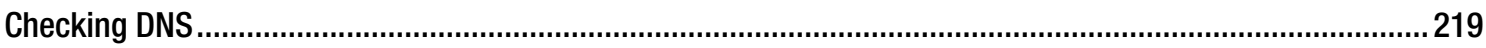

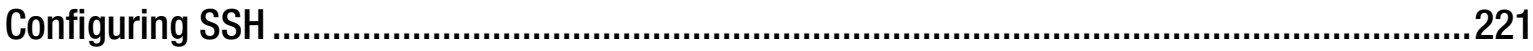

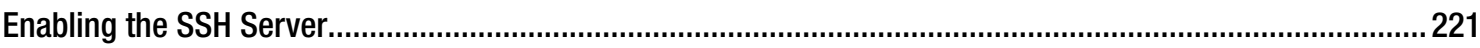

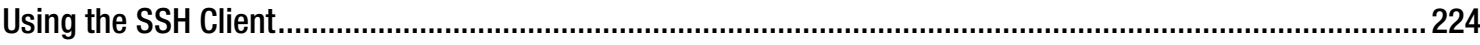

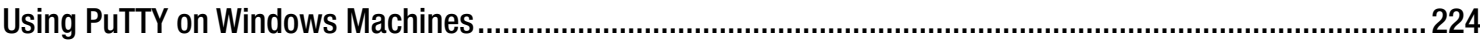




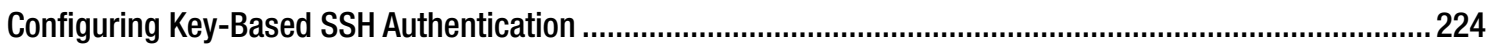

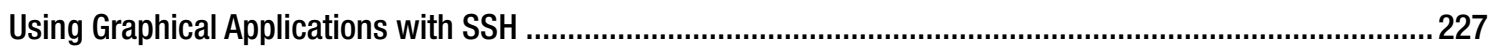

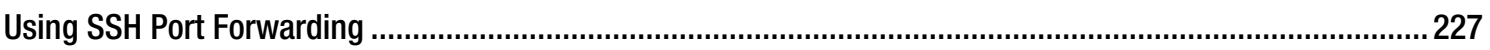

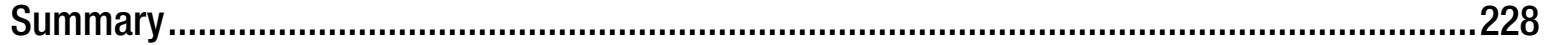

Chapter 10: Securing Internet Services: Certificates and SUSE Firewall .....................229

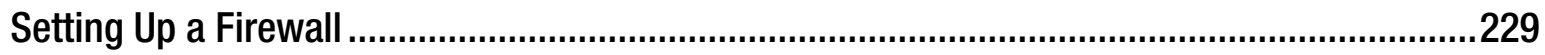

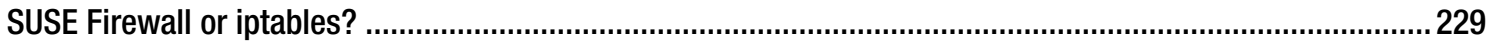

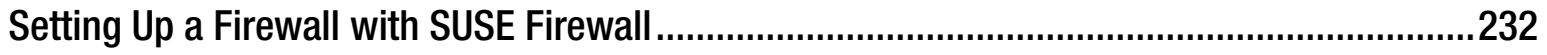

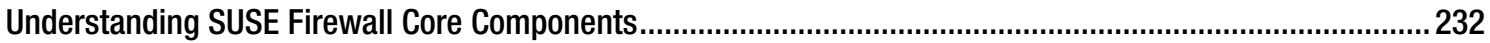

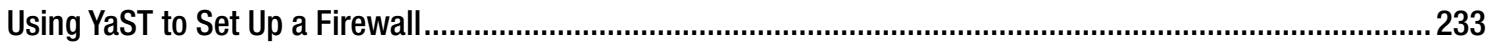

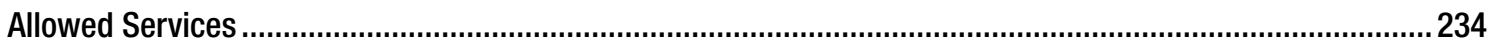

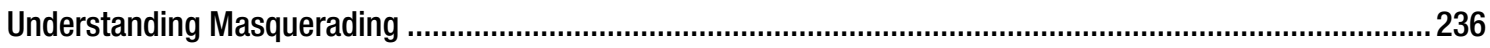

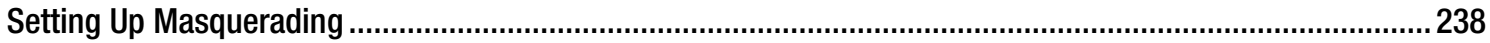

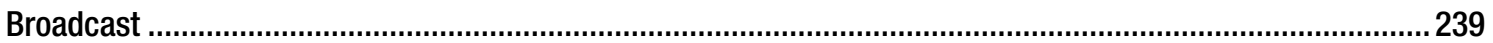

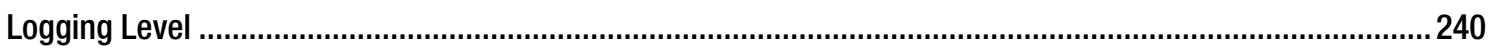

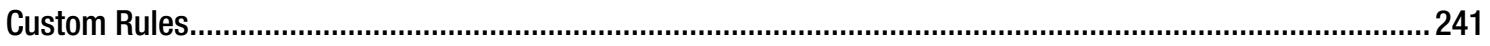

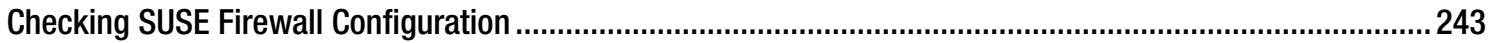

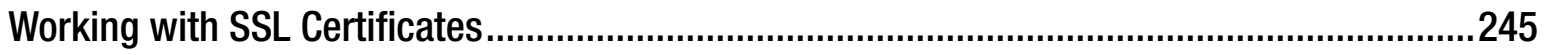

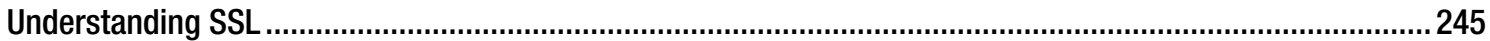

Configuring the YaST Certificate Authority........................................................................................... 247

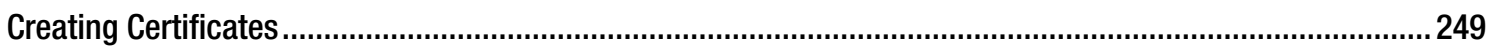

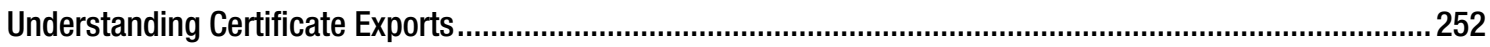

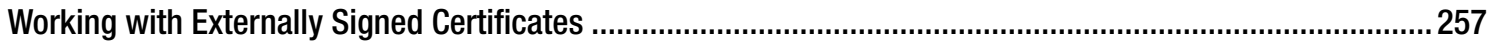

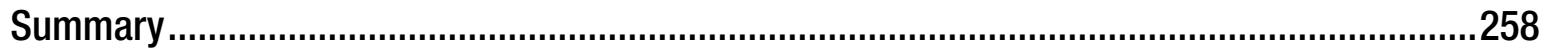

Chapter 11: Basic Network Services: xinetd, NTP, DNS, DHCP, and LDAP ....................259

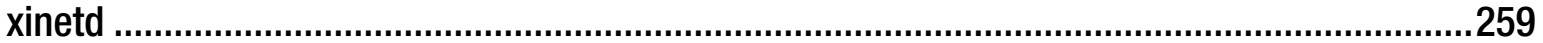

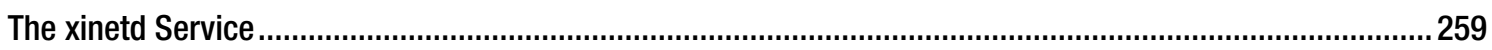

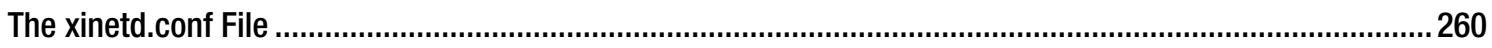

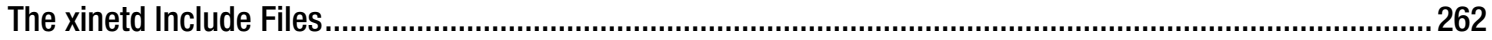


NTP 262

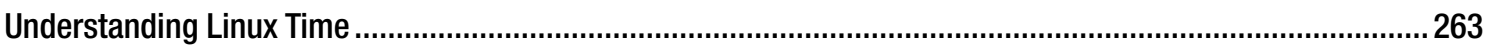

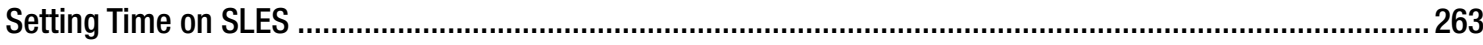

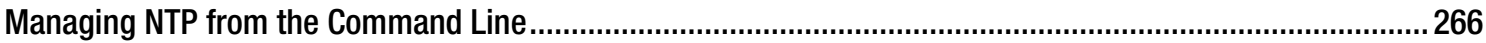

Using ntpq and ntpdc for NTP Server Management ............................................................................ 268

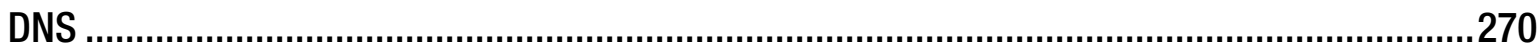

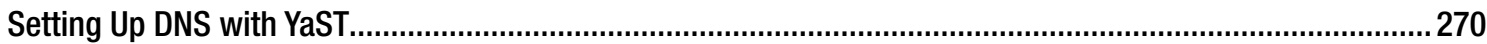

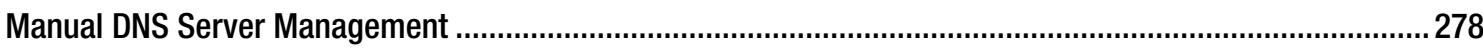

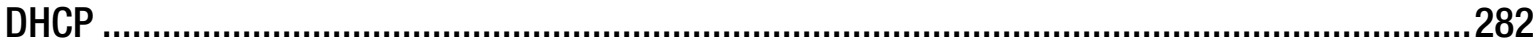

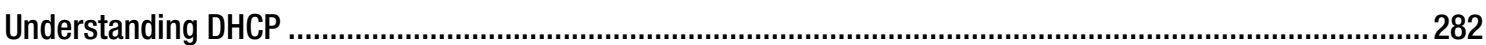

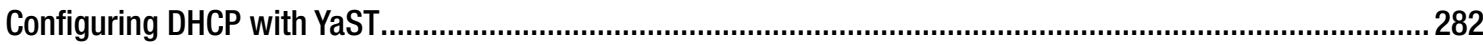

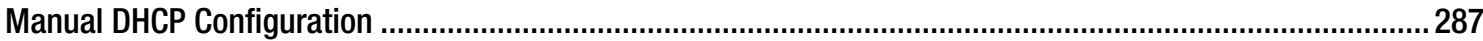

Monitoring and Testing DHCP Functionality.................................................................................... 288

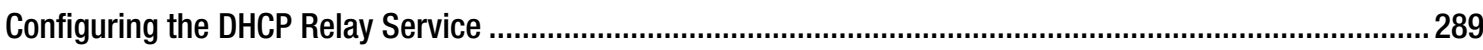

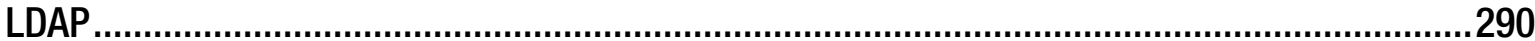

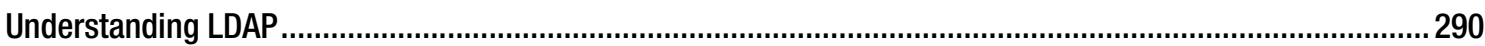

Setting Up an LDAP Server with YaST...............................................................................................291

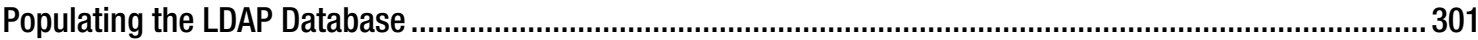

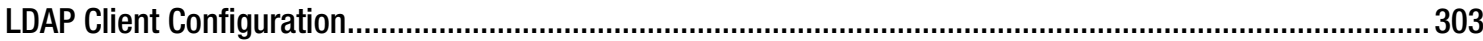

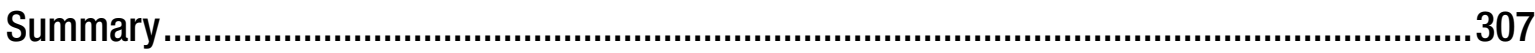

Chapter 12: Setting Up a LAMP Server ....................................................................309

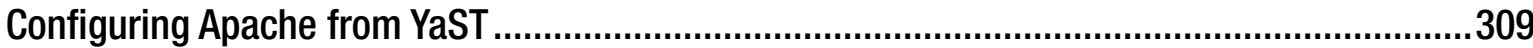

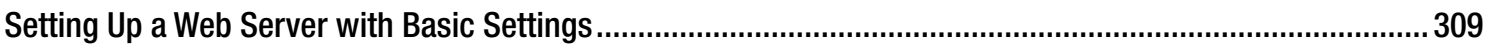

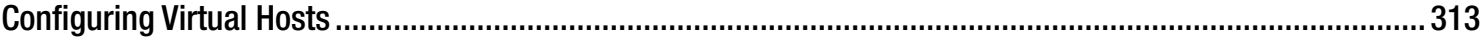

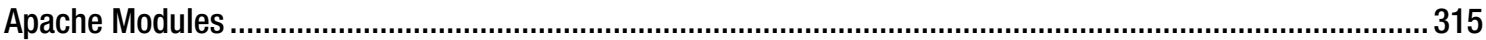

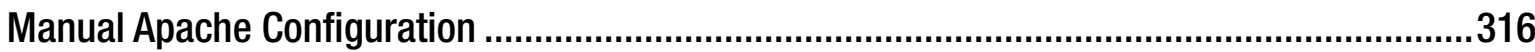

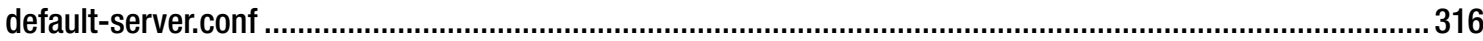

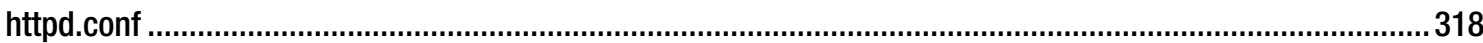




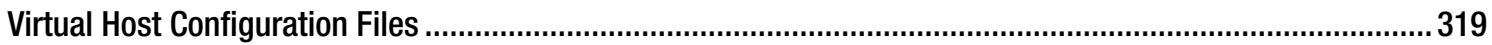

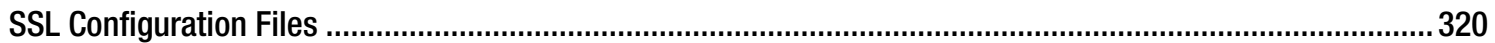

Setting Up Authentication .................................................................................................................. 320

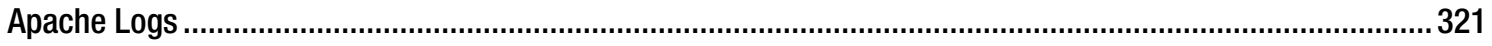

Configuring the Database Part of the LAMP Server ..........................................................322

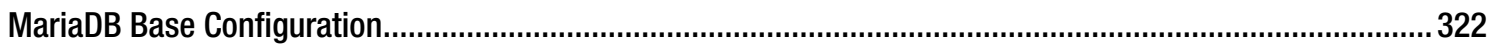

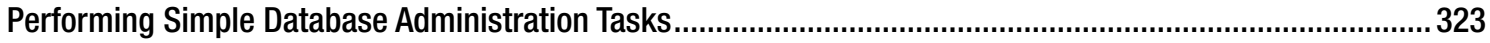

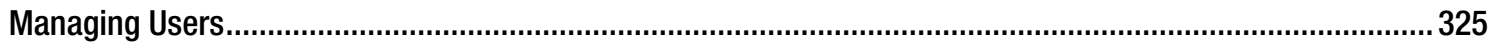

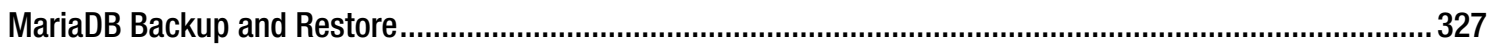

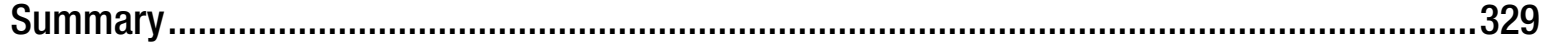

Chapter 13: File Sharing: NFS, FTP, and Samba .............................................................331

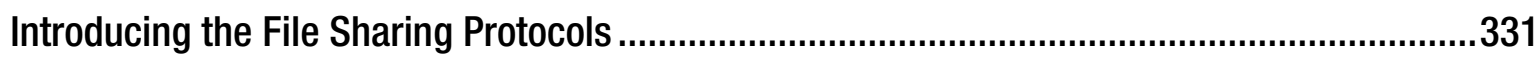

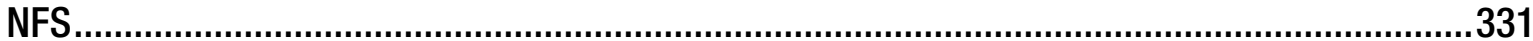

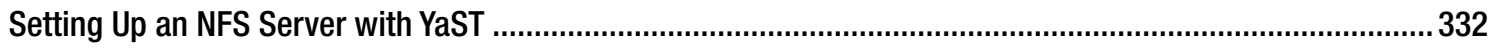

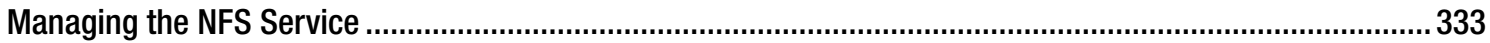

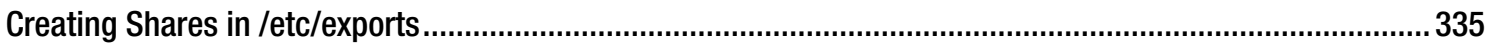

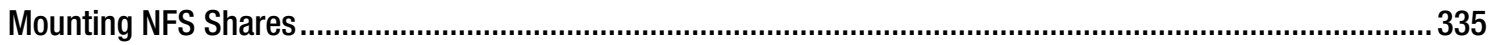

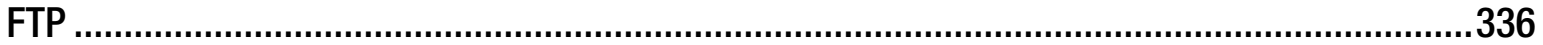

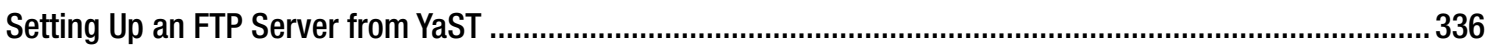

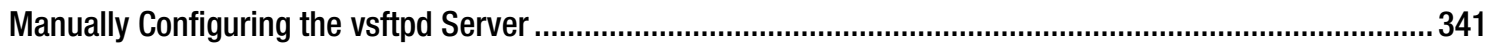

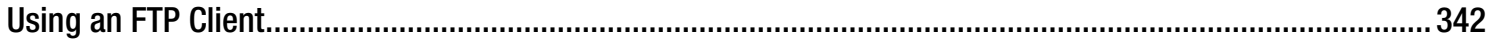

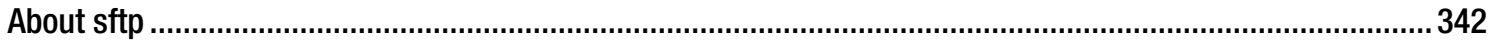

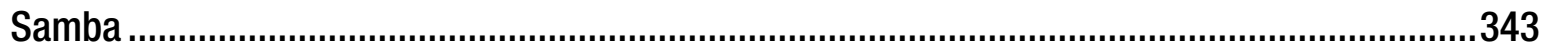

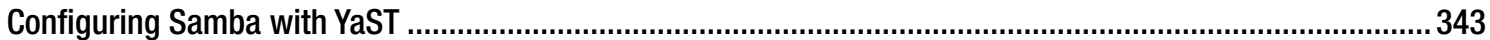

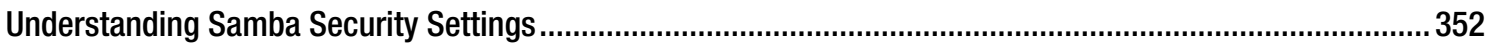

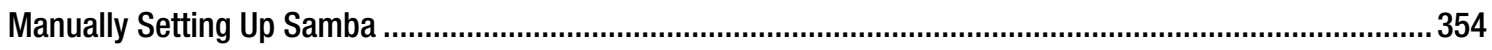

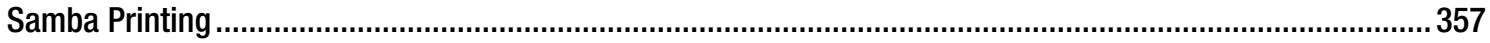

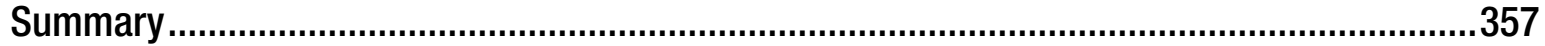




\section{Part IV: Advanced SUSE Linux Enterprise Server Administration.............. 359}

Chapter 14: Introduction to Bash Shell Scripting .......................................................361

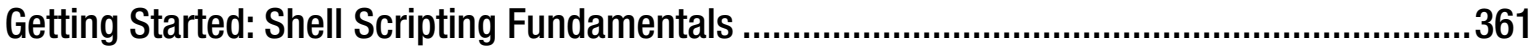

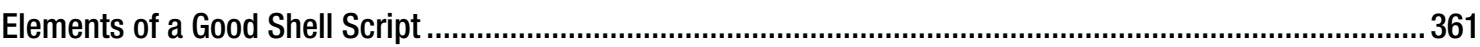

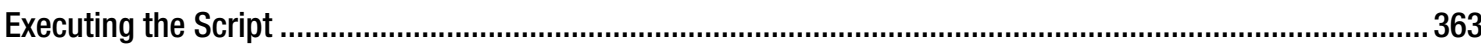

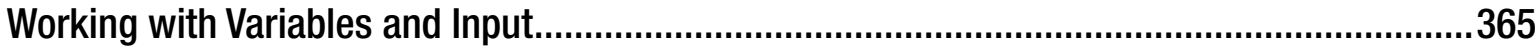

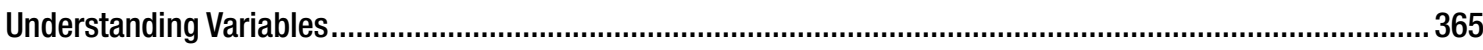

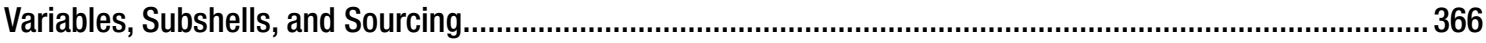

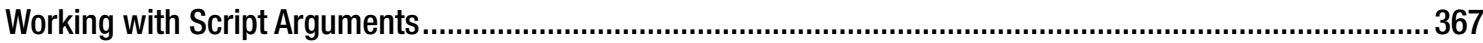

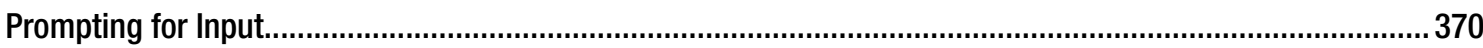

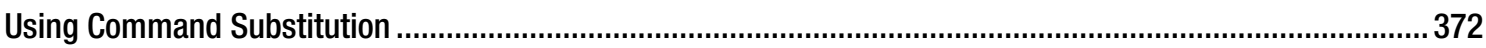

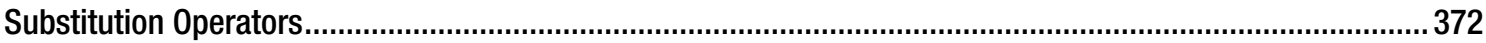

Changing Variable Content with Pattern Matching ................................................................................ 374

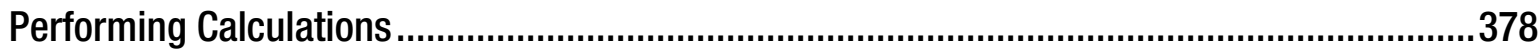

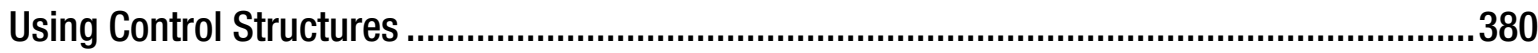

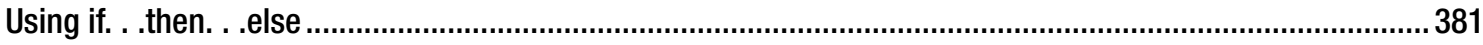

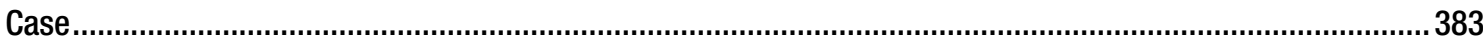

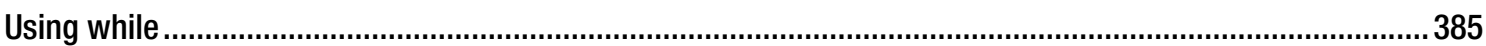

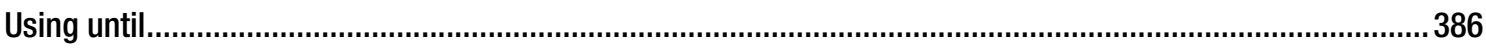

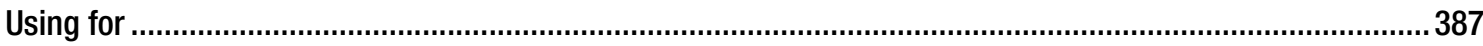

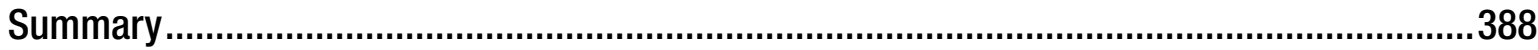

Chapter 15: Performance Monitoring and Optimizing .................................................389

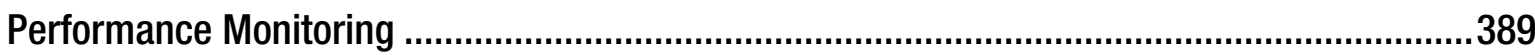

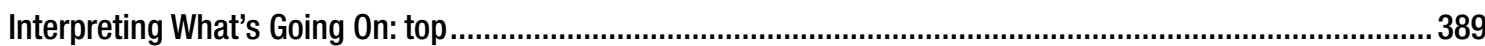

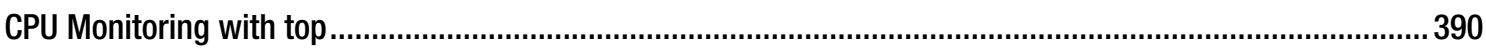

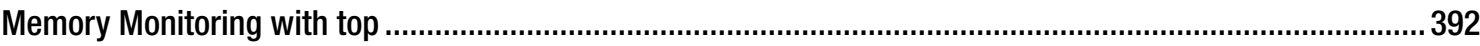

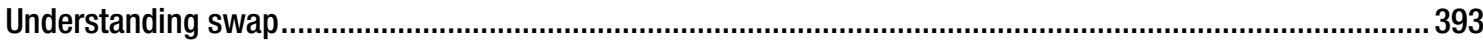

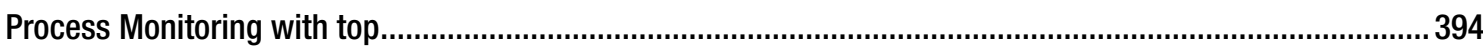




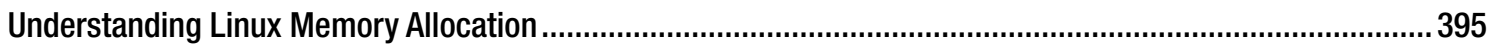

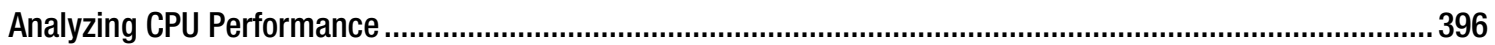

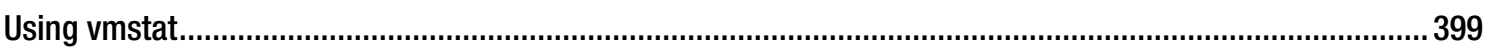

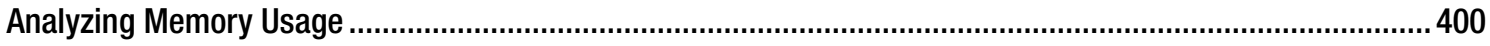

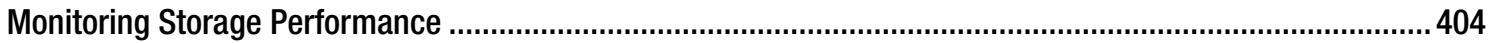

Understanding Network Performance ................................................................................................ 409

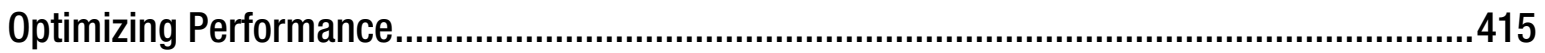

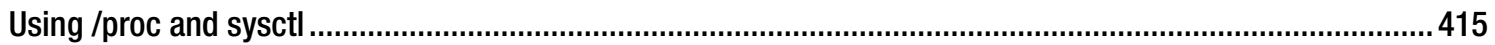

Using a Simple Performance Optimization Test.................................................................................... 416

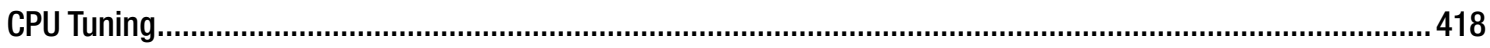

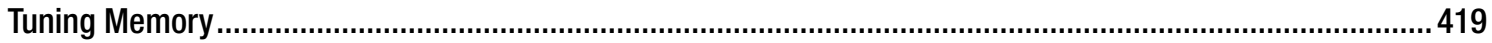

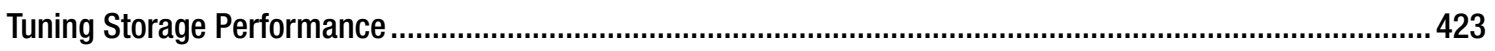

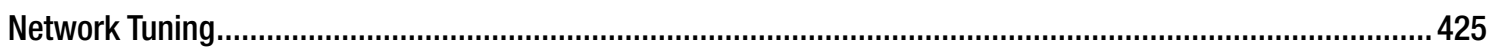

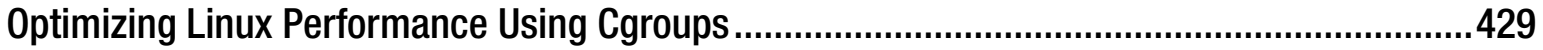

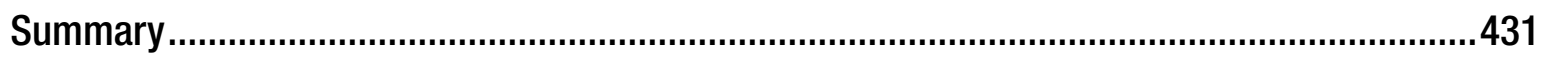

Chapter 16: Creating a Cluster on SUSE Linux Enterprise Server...............................433

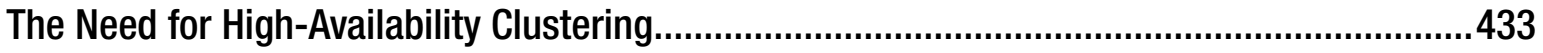

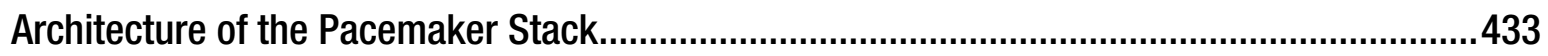

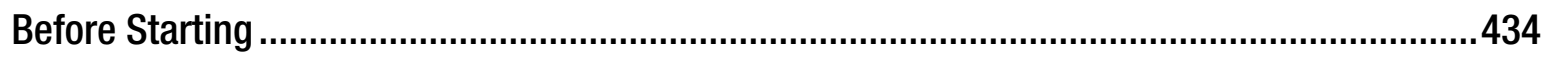

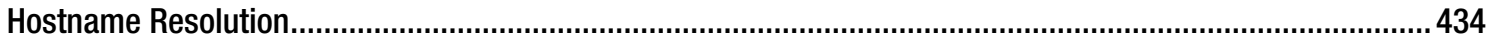

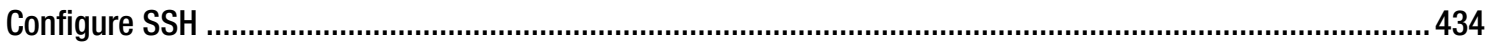

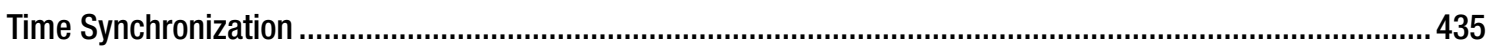

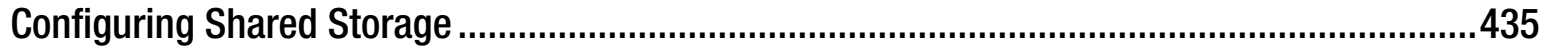

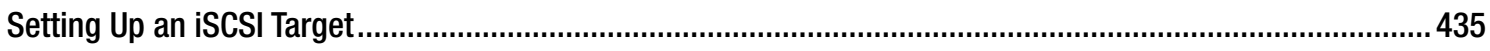

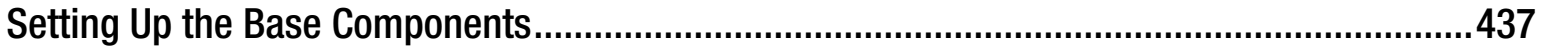

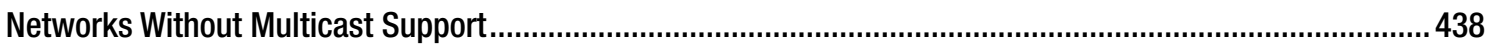

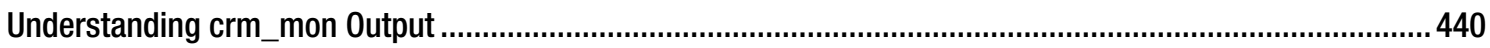




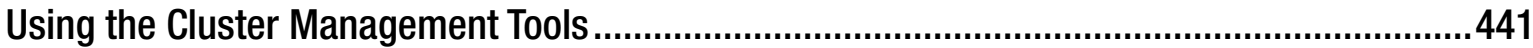

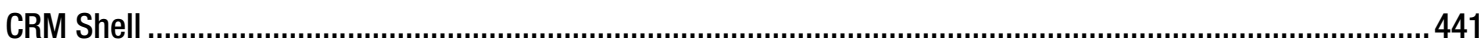

Hawk

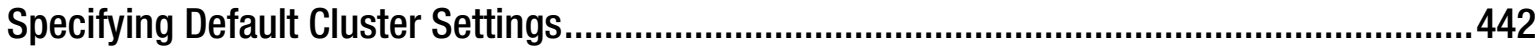

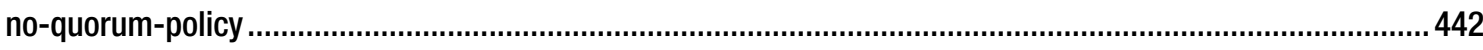

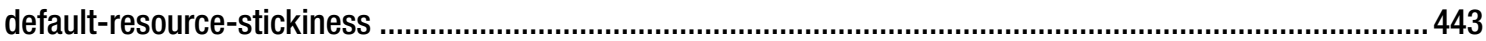

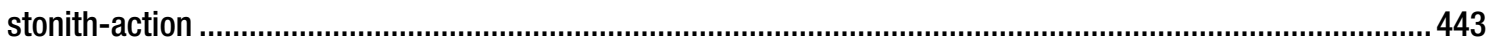

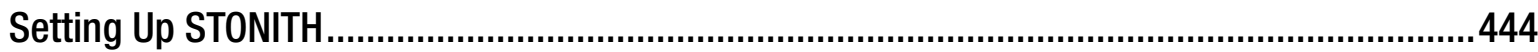

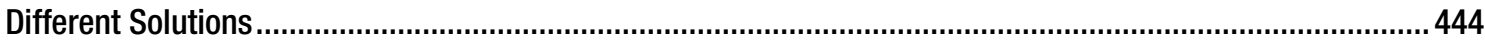

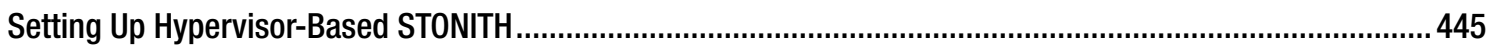

Setting Up Shared Disk-Based STONITH.............................................................................................. 447

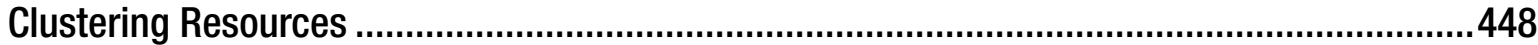

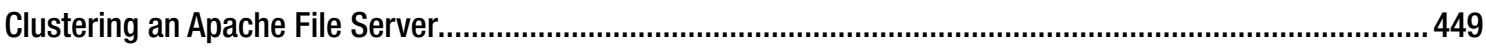

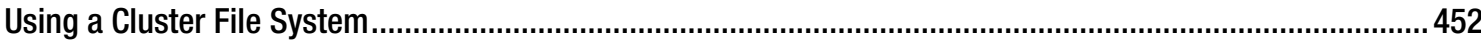

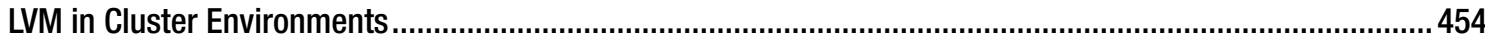

Fine-Tuning the Cluster with Constraints ................................................................................................. 457

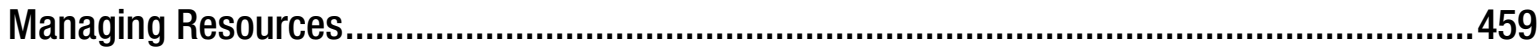

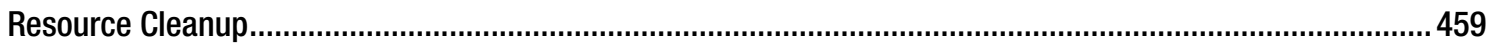

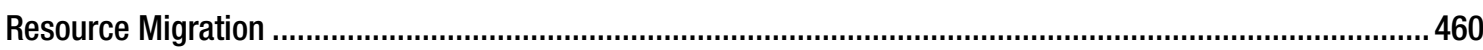

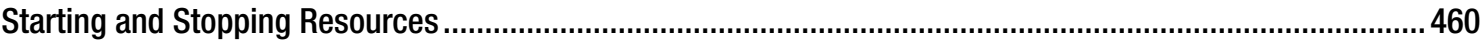

Using Unmanaged State for Maintenance ............................................................................................. 460

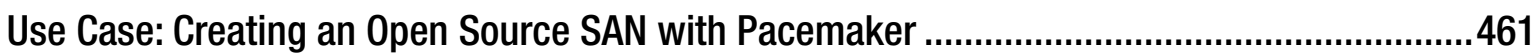

Configuring RAID 1 over the Network with DRBD .................................................................................. 461

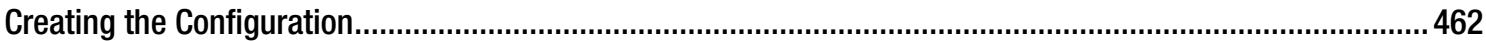

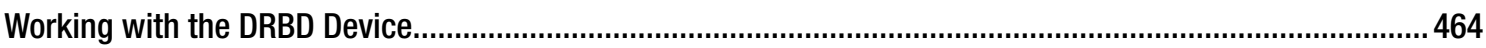

Troubleshooting the Disconnect State ....................................................................................................... 464

Working with Dual Primary Mode ............................................................................................................. 465

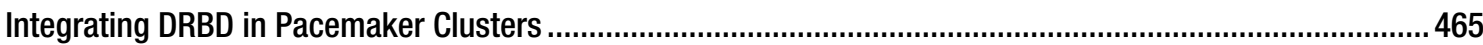




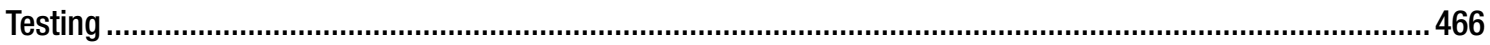

Adding an iSCSI Target to the Open Source SAN .................................................................................. 466

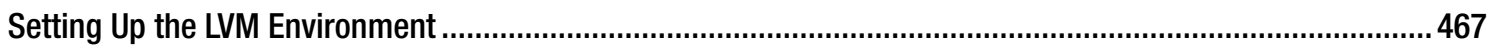

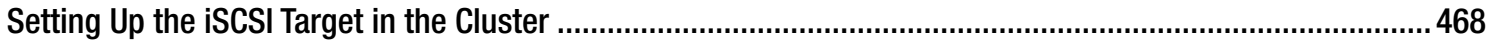

Summary

Chapter 17: Creating a SLES 12 Installation Server ..................................................471

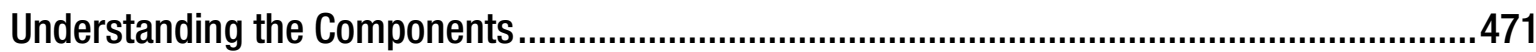

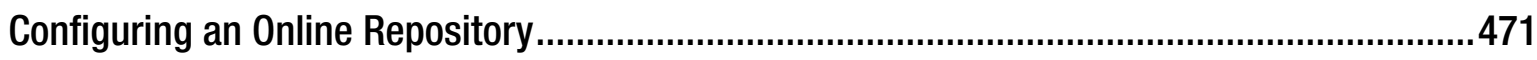

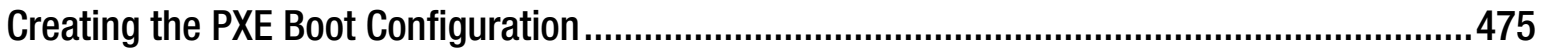

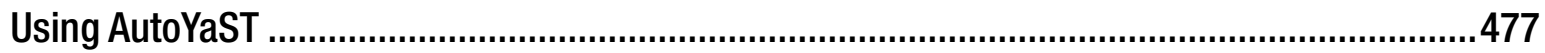

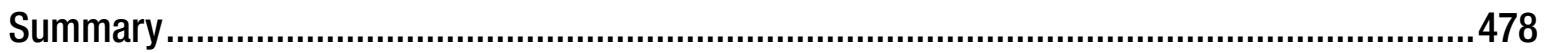

Chapter 18: Managing SUSE Linux .............................................................479

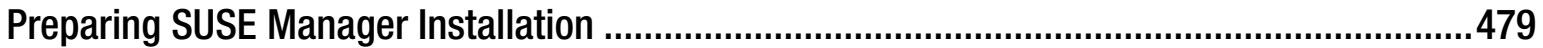

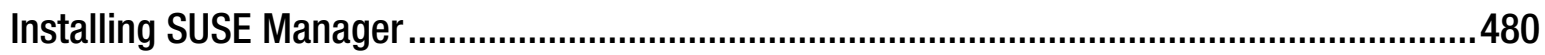

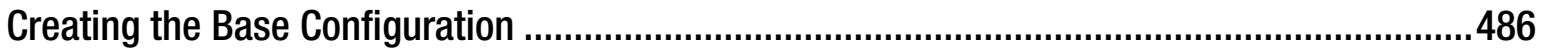

Using the Setup Wizard from the Web Console ................................................................................... 486

Managing Package Synchronization........................................................................................................ 488

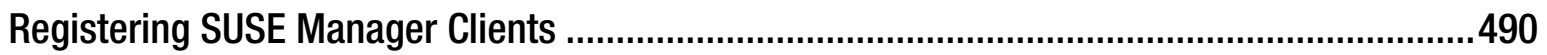

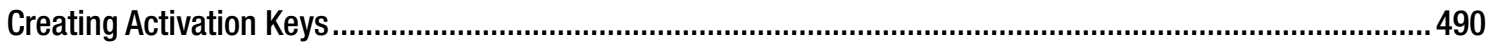

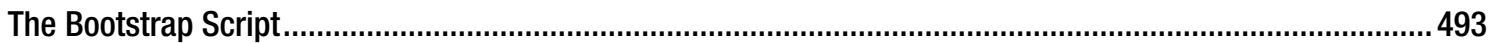

Troubleshooting and Unregistering Registered Systems........................................................................ 496

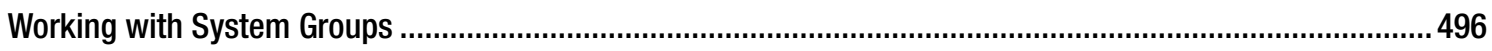

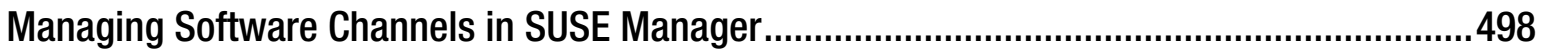

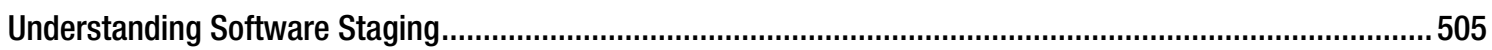

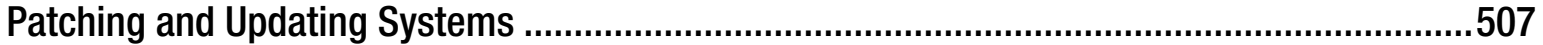

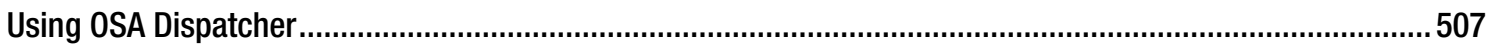




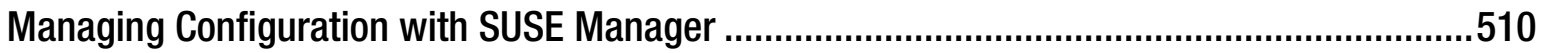

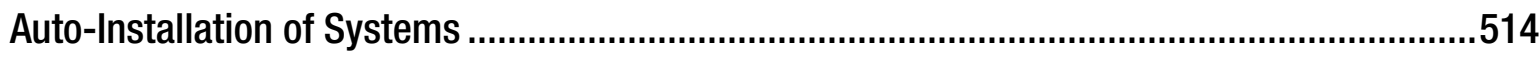

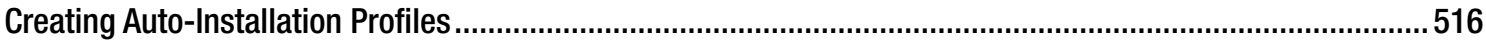

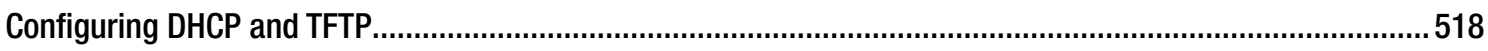

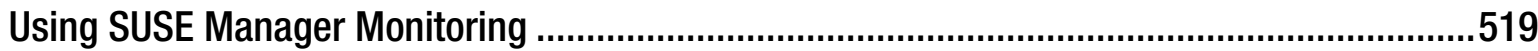

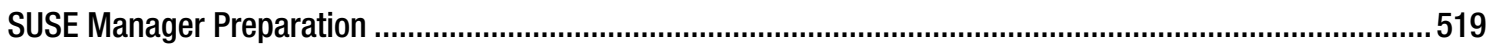

Preparing the Registered Servers for Monitoring ................................................................................ 522

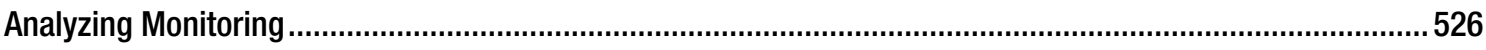

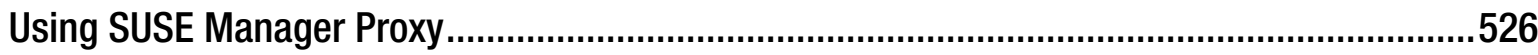

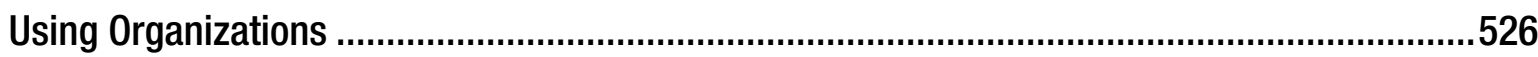

Summary

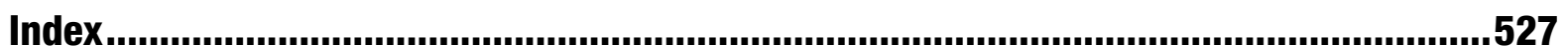




\section{About the Author}

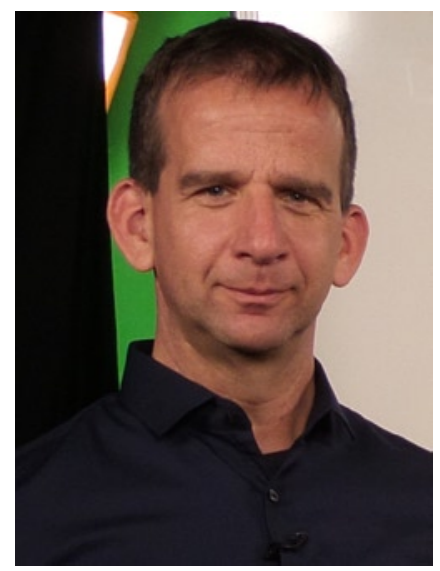

Sander van Vugt is a Linux expert working from the Netherlands as an author, technical trainer, and consultant for clients around the world. Sander has published several books about different Linux distributions and is a regular contributor to major international Linux-related web sites. As a consultant, he is specialized in Linux high availability and performance optimization. As a technical trainer, Sander is an authorized trainer for SUSE Linux Enterprise Server and Red Hat Enterprise Linux. More information about the author can be found at his web site at wwW. sandervanvugt. com. 



\section{About the Technical Reviewer}

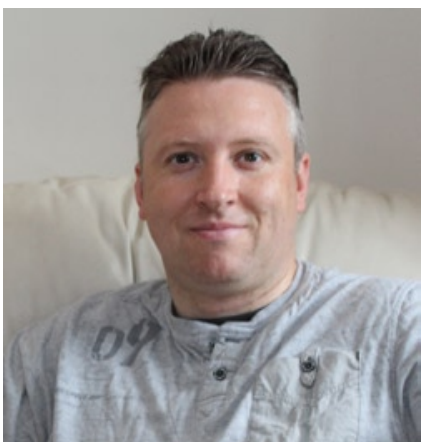

Stewart Watkiss graduated from the University of Hull, UK, with a master's degree in electronic engineering. He has been a Linux user since first installing it on a home computer during the late 1990s. Stewart has worked in the IT industry for 18 years, including positions in computer networking, IT security, and as a Linux system administrator.

While working as a Linux system administrator, he was awarded Advanced Linux Certification (LPIC 2) in 2006 and Novell Certified Linux Administrator for SUSE Linux Enterprise 11 in 2010. He created the PenguinTutor web site to help others learning Linux and working toward Linux certification (www. penguintutor. com).

Stewart also volunteers as a STEM ambassador, going into local schools to help support the teachers and to teach programming to teachers and children. 



\section{Acknowledgments}

First, I want to thank Dominic Shakeshaft and Michelle Lowman, for their willingness to publish this book. I also want to thank Kevin Walter, who has helped me throughout the long road that has lead to the realization of the first draft of all chapters being ready, even before the final software was realized. This is the 59th book I have written and the very first time that the rough version of the book was ready before the software! Kevin, if you had not pushed me at the right moments, this would never have happened! I also want to thank technical editor Stewart Watkiss and Michael G. Laraque for their excellent work, which has helped improve the quality of this book.

Apart from the people at Apress, I'd like to thank those at SUSE who have helped me get the software and information I needed to realize the book. A special thanks to Matthias Eckermann, product manager of SLES, who has played an important role in this process, and to Gábor Nyers, who provided me with excellent information that has lead to the chapter about SELinux.

I also want to thank the people at Kiabi in Lille, France. It was while instructing them in early 2014 that I got the inspiration to start writing the chapter about high availability, which motivated me to add 17 other chapters as well, which has lead to the book in front of you.

Finally, I want to thank my son Franck for helping with the line art in this book. Keep on doing that, and I'm sure you'll be a great graphic artist! 



\section{Introduction}

This book is about SUSE Linux Enterprise Server 12. It is intended for readers who already have basic Linux skills, so you won't find information on how to perform really basic tasks. Some elementary skills are briefly explained, after which, in a total of 18 chapters, the specifics of working with SUSE Linux Enterprise Server are touched upon.

While writing this book, I have decided it should not be just any generic Linux book that happens by accident to be about SUSE Linux Enterprise Server. Instead, I have focused on those tasks that are essential for Linux professionals who need to know how specific tasks are performed in an SUSE environment. That is why the SUSE administration tool YaST plays an important role in this book. YaST was developed to make administering SUSE Linux easy. In previous versions of SUSE Linux, YaST had a bad reputation, as on some occasions, it had overwritten configurations that the administrator had carefully built manually. On SUSE Linux Enterprise Server (SLES) 12 that doesn't happen anymore, and that is why YaST provides an excellent tool to build the basic configurations that are needed to do whatever you want to do on your Linux server. That is why many chapters begin with an explanation of how tasks are accomplished through YaST.

I am also aware, however, that using YaST alone is not sufficient to build a fully functional SLES server. That is why after explaining how to accomplish tasks with YaST, you'll learn which processes and configuration files are used behind them, which allows you to manually create the exact configuration you require to accomplish whatever you need to accomplish on your server.

As I am a technical trainer myself, I have also included exercises throughout this book. These exercises help readers apply newly acquired skills in SLES and also help those who are preparing for the SUSE CLA and CLP exams. I have not written this book as a complete course manual for these exams, however, although it will serve as an excellent guide to preparing for these exams.

This book is organized in four different parts. The first parts briefly touch on basic skills. In Chapter 1, you'll learn how SUSE relates to other Linux distributions, and Chapter 2 covers the SUSE Linux Management basics. In this chapter, you'll learn how YaST is organized and what you can do to make the best possible use of it.

The second part is about Linux administration basics. You'll first learn about file systems, including the new Btrfs file system and its features, in Chapter 3. Following that, you'll learn how to create users, configure permissions, apply common tasks, and harden SLES. The last two chapters in this section are about virtualization and management of hardware, the kernel, and the boot procedure, which includes the new systems process that takes care of everything that happens while booting.

The third part is about networking SLES. You'll learn how to use the new wicked tool to configure networking and how to set up essential services that are used in a network context, including firewalling, SSL managing, DNS, DHCP, LDAP, LAMP, NFS, and FTP. This section should help you get going, no matter which network services you want to configure.

The fourth and final part of this book is about advanced administration tasks. You'll learn how to write and read shell scripts, how to optimize performance, how to build a high-availability cluster, how to configure an installation server, and how to manage SUSE Linux using SUSE Manager. 